Journal of Social Sciences (COES\&RJ-JSS)

ISSN (E): 2305-9249 ISSN (P): 2305-9494

Publisher: Centre of Excellence for Scientific \& Research Journalism, COES\&RJ LLC

Online Publication Date: $1^{\text {st }}$ April 2019

Online Issue: Volume 8, Number 2, April 2019

https://doi.org/10.25255/jss.2019.8.2.279.291

\title{
Civil State and its impact on reform
}

Dr. Tammam Odeh Abdullah Al Assaf

PhD. in Islamic Jurisprudence and Principles

Associate Professor

Faculty of Sharia

University of Jordan

\begin{abstract}
:
Islam sanctions and affirms the openness of Muslim society to the civilizational outputs of other societies without any a priori sensitivities. This is grounded in digesting and assimilating the civilizational outputs transfused into Islamic civilization from another cultural environment, and indigenizing them in an Islamic cultural environment. The final outcome derived from applying Religion is the establishment of social existence and life on the basis of the guidance of Religion, or what the latter was mute about in the affairs of life which are left to human endeavor. This is in essence what the Islamic state is about: it is a political entity formed by Muslims based on Religion. Emphatically it is not a meta-human state, for it derives what it can from Religion, while leaving up to Muslims to introduce what religion was silent about in the quest to conduct the affairs of the state. The transformation to a state of laws and humanity is in need of the political will which is inclined to overcoming all the obstacles and problems preventing the establishment of the civil state, the liberation of civil society, and removing the restrictions on its activity and efficacy.
\end{abstract}

\section{Keywords:}

Civil state, democracy, secularism, reform

\section{Citation:}

Al Assaf, Tammam Odeh Abdullah (2019); Civil state and its impact on reform; Journal of Social Sciences (COES\&RJ-JSS), Vol.8, No.2, pp:279-291; https://doi.org/10.25255/jss.2019.8.2.279.291. 


\section{الاولة المدنية وأثرها في الإصلاح}

Dr. Tammam Odeh Abdullah Al Assaf

د. دمام عودة عبدالله العساف

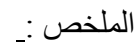

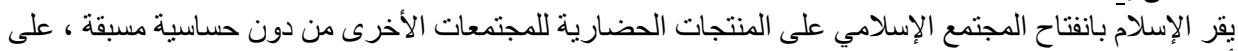

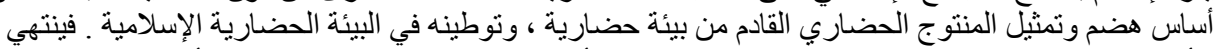

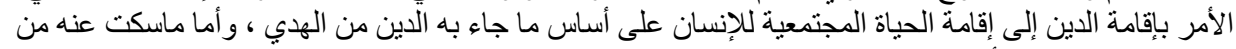

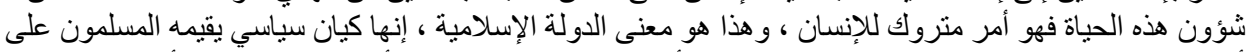

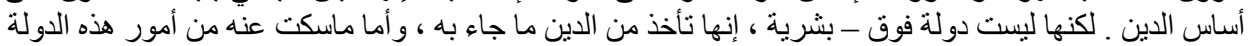

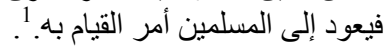

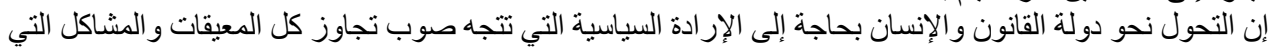

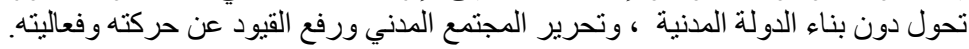

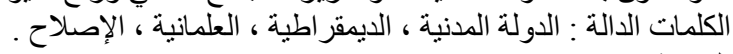

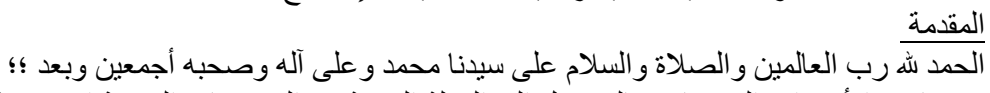

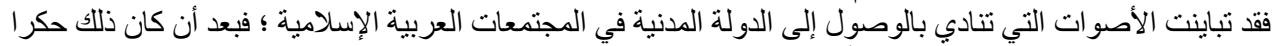

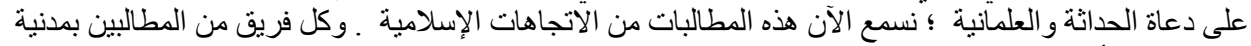

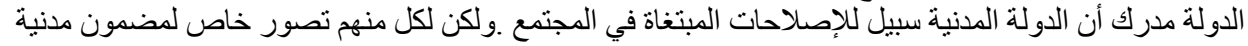

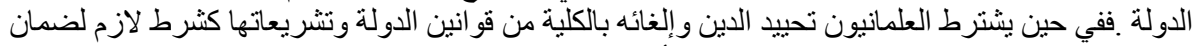

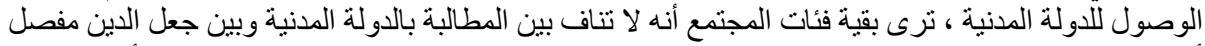

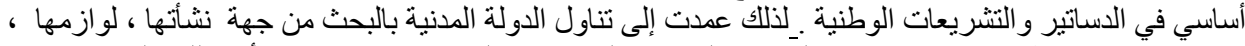

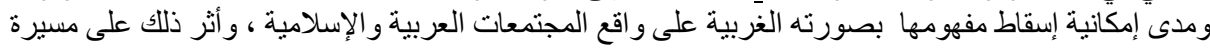
الإصناح الحماح

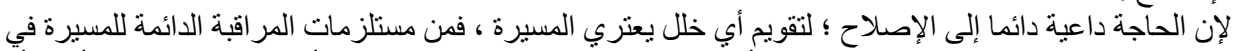

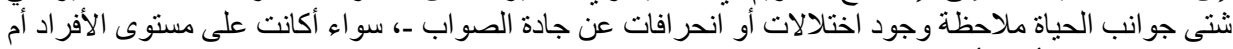

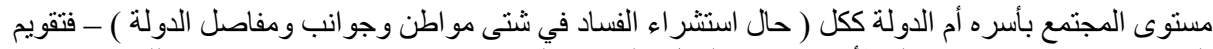

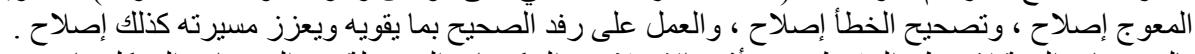

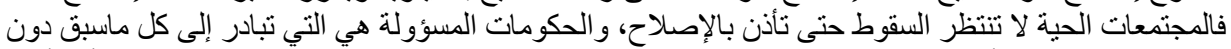

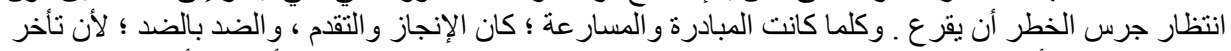

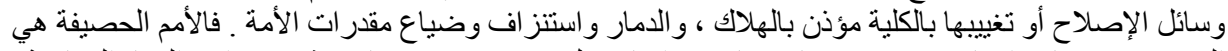

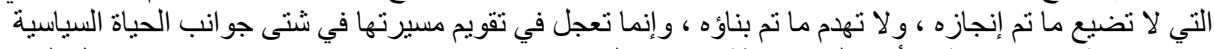

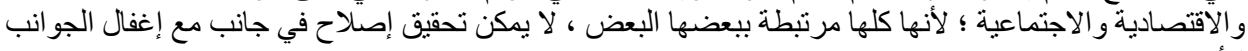

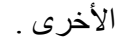

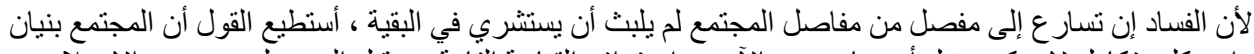

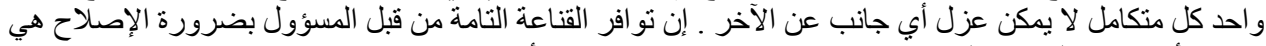

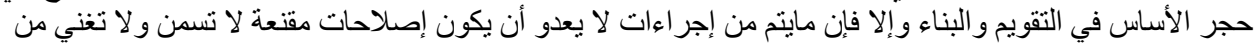

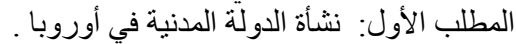

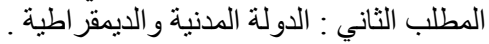

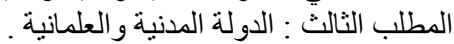
المطلب الر ابع : الدولة المدنية في الواقلة المدانية الإسلامي.

1 انظر : عبد الجبار محمد ، الإسلام و الديمقر اطية في معركة البناء الحضاري ، ص130. 


\section{Civil state and its impact on reform}

المطلب الأول : نشأة الدولة المدنية في أوروبا.

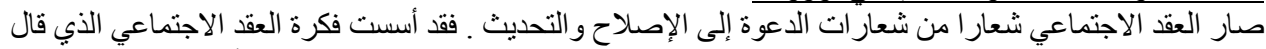

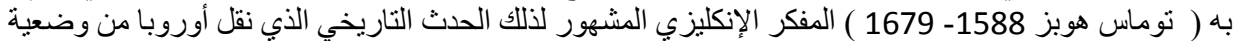

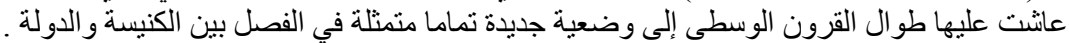

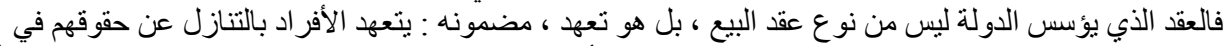

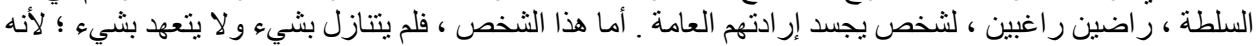

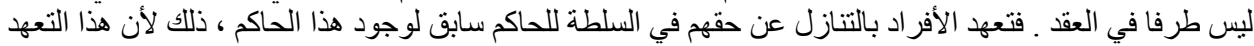

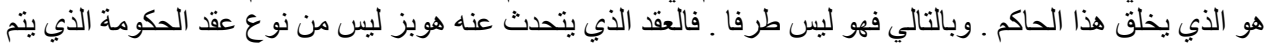

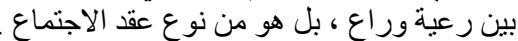

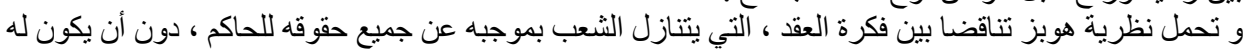

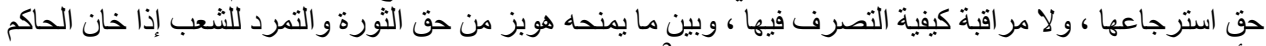

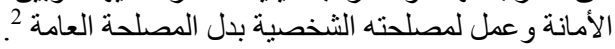

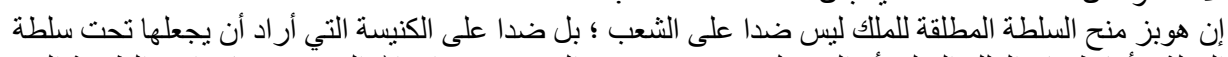

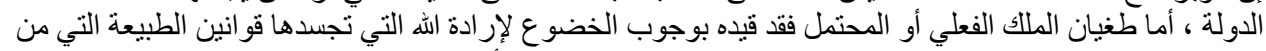

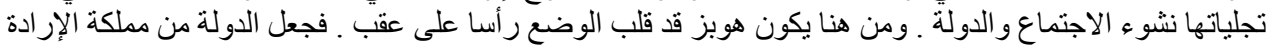

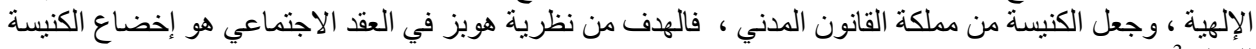

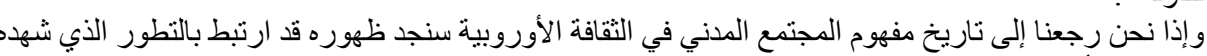

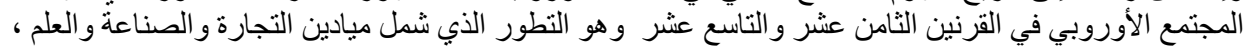

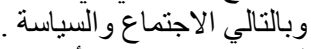

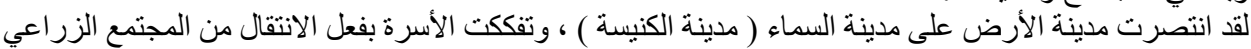

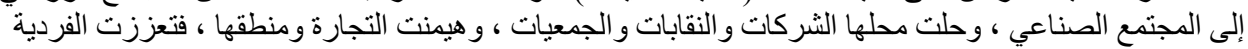

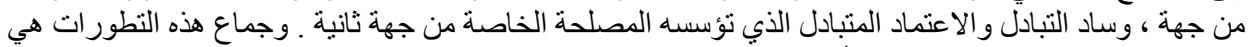

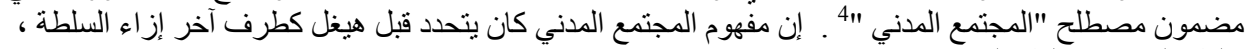
سلطة الكنيسة وسلطة الحكم الاستبدادي .

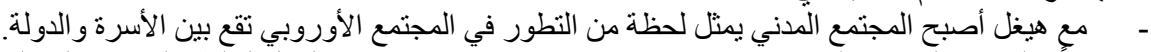

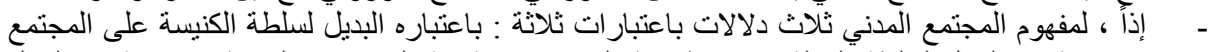

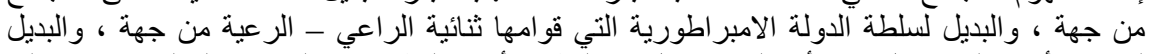
كلهيمنة الأسرة التي تتثنل في الأب الذي ينحول في النظام الأبوي البطريركي إلى شيخ القبيلة من جهة ثنالثة

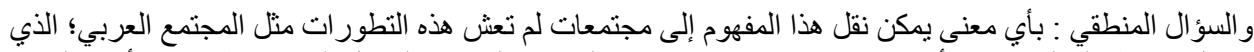

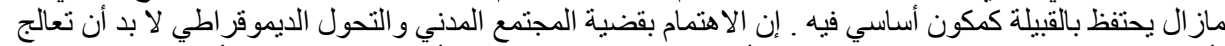

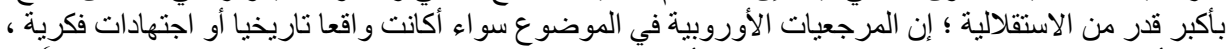

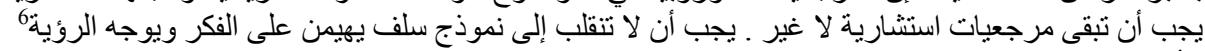

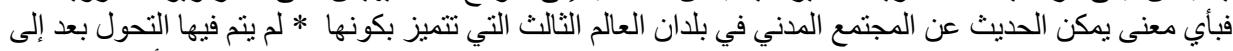

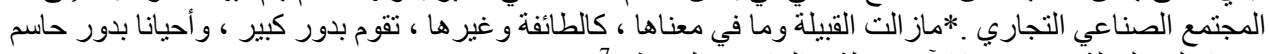

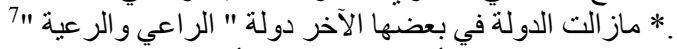

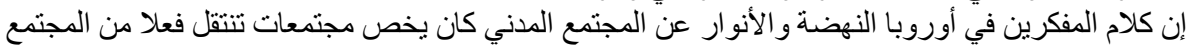

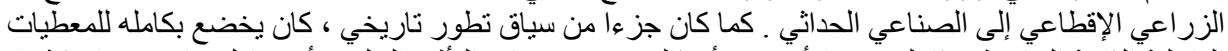

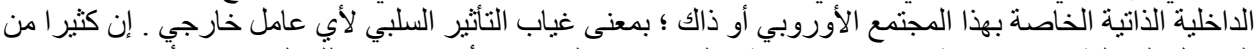

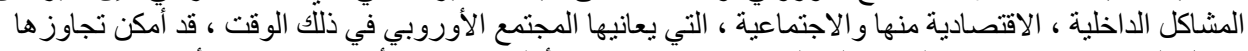

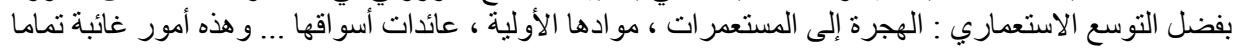

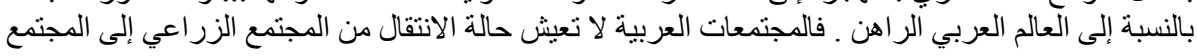

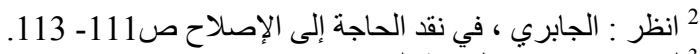

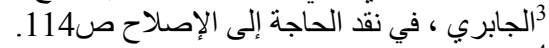

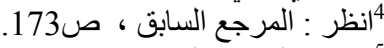

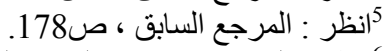

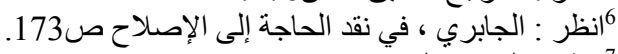

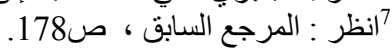




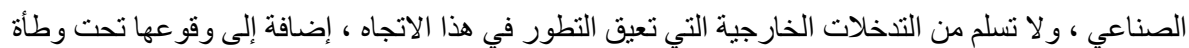

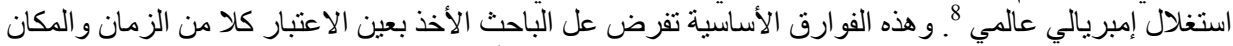

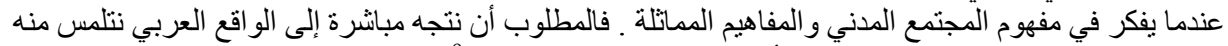

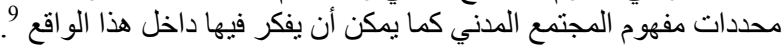

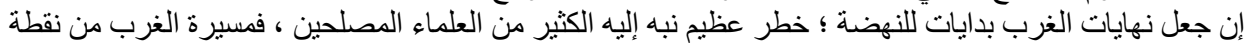

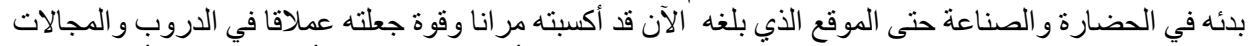

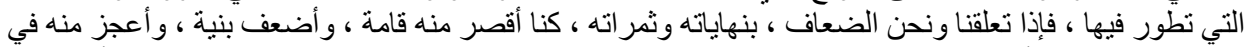

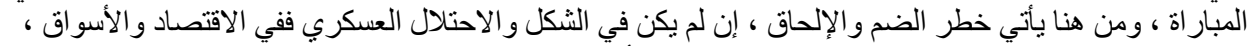

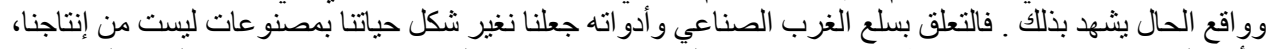

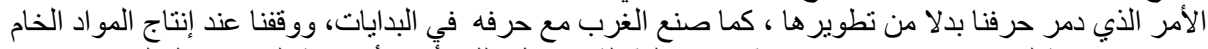

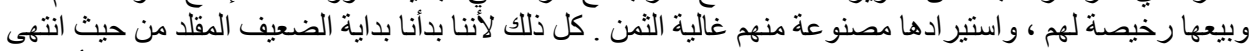

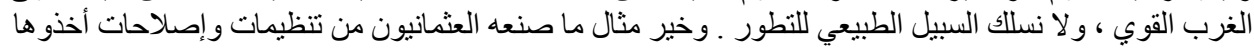

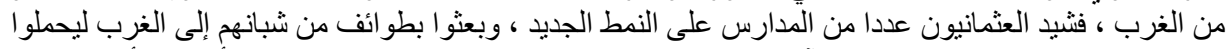

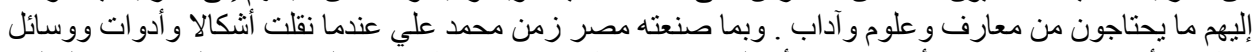

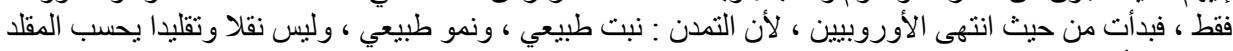

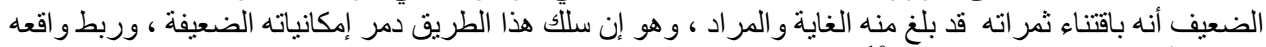

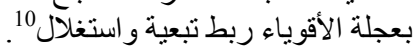
المطلب الثاني : الدولة المدنية و الدئية الديمقر اطية.

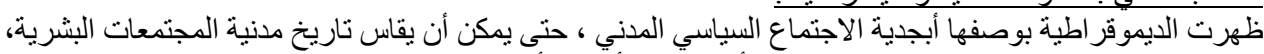

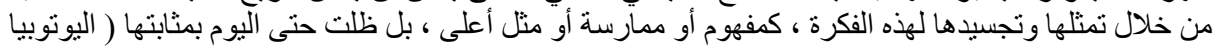

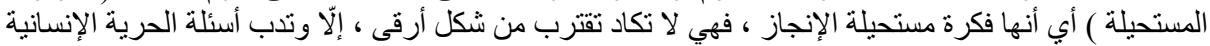

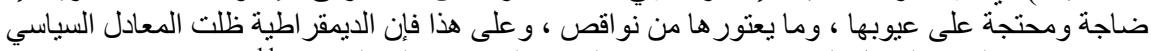

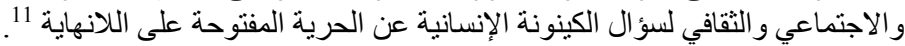

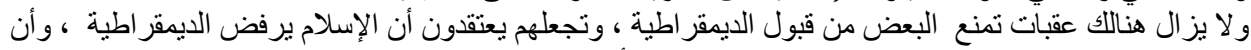

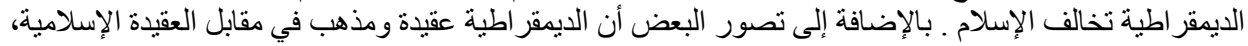

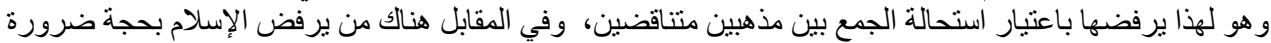

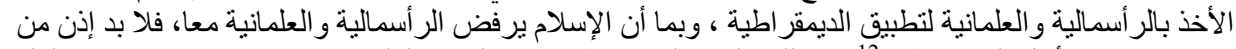

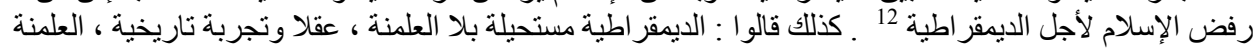

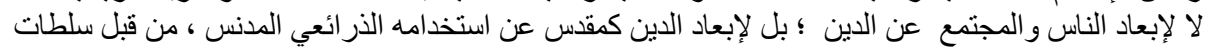

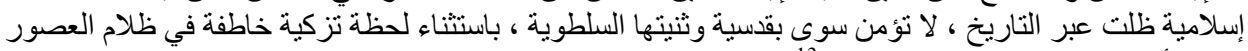

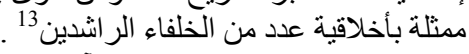
ويجلي هذا الفهم الخاطئ الحقائق الآتية : الفين

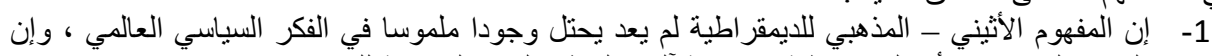

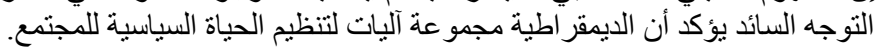

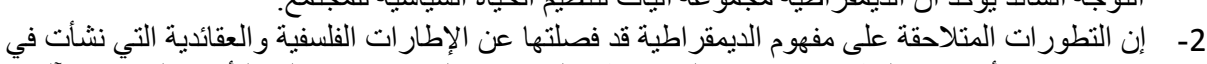

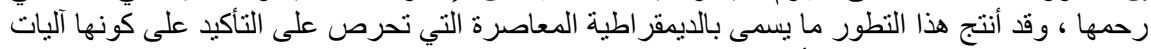

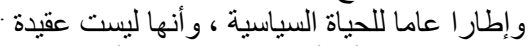

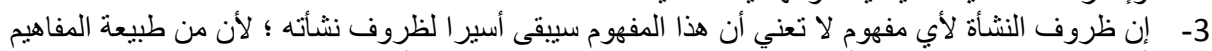

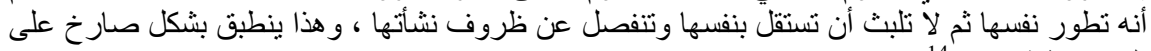

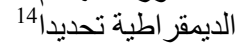

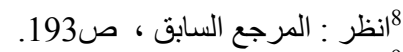

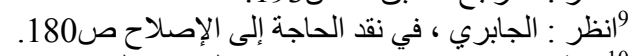

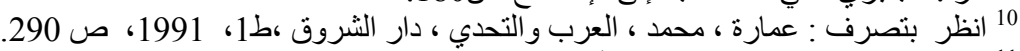

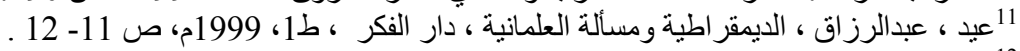

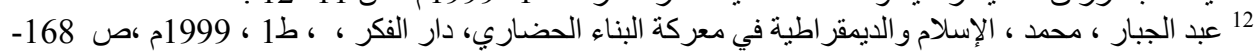




\section{Civil state and its impact on reform}

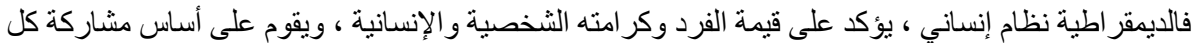

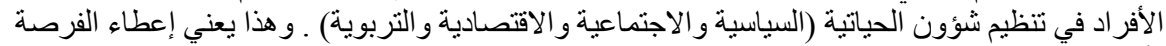

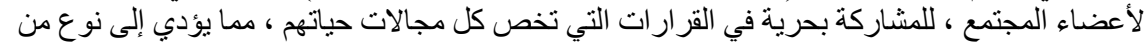

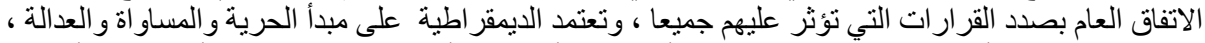

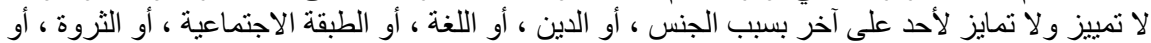

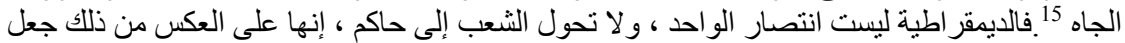

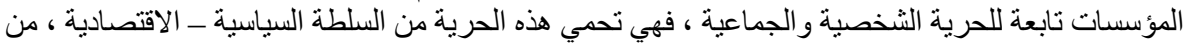

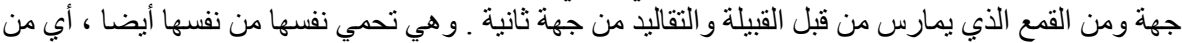

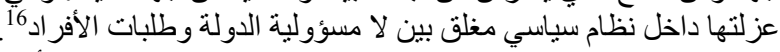

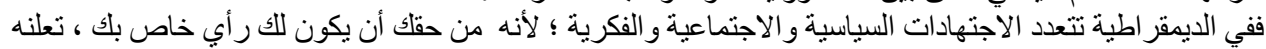

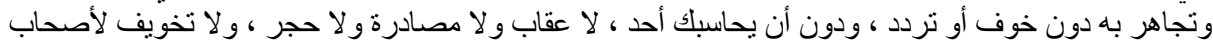

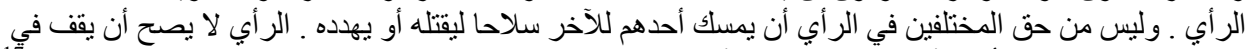

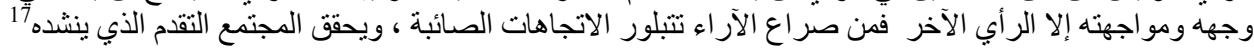

إن تطبيق أو تعزيز الديمقر اطية ينطلب شروطا يجب نو افرها مثل الو الوعي السياسي ، المستوى التعليمي ، وتطور

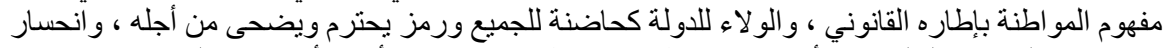

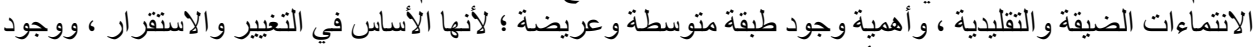

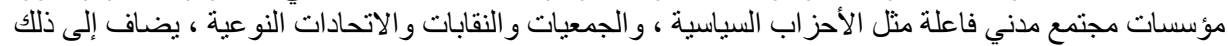

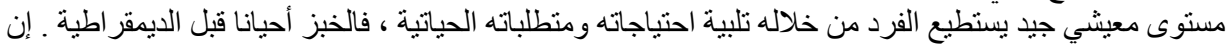

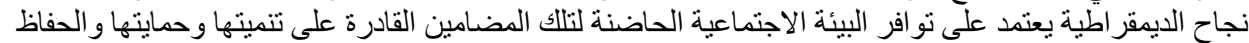

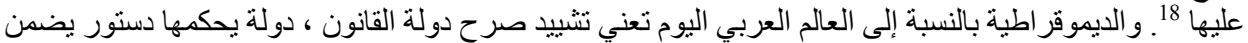

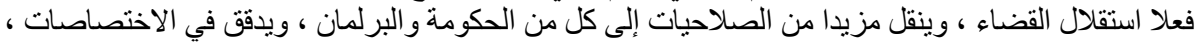

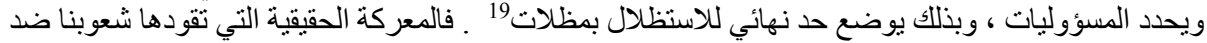

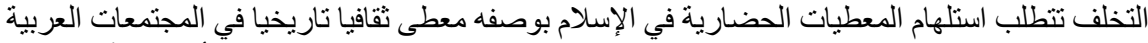

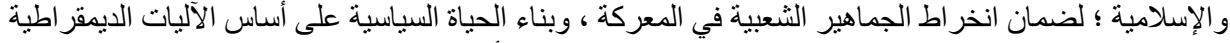

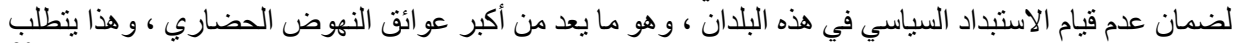

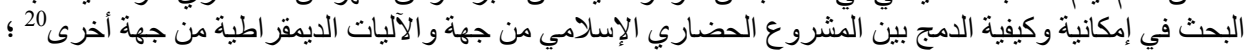

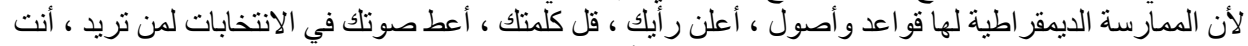

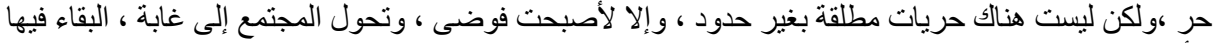

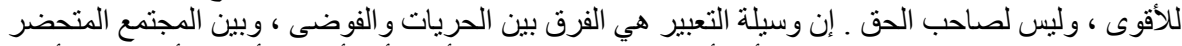

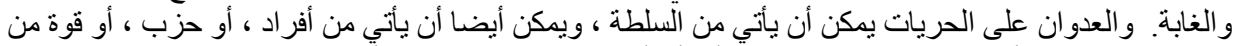

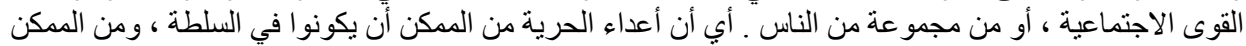

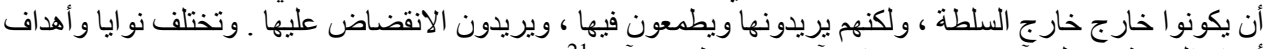

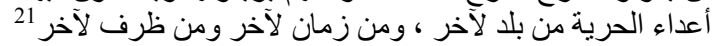

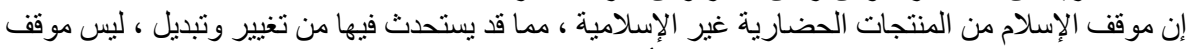

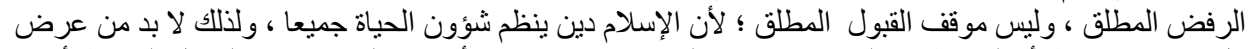

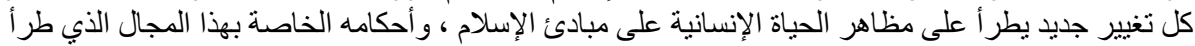

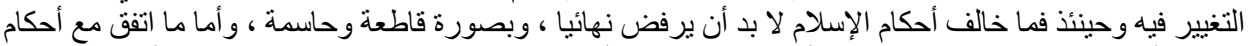

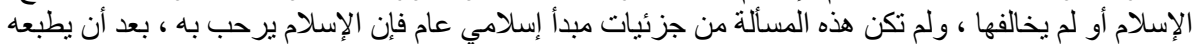

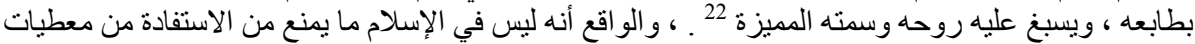

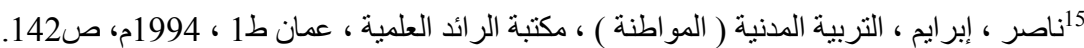

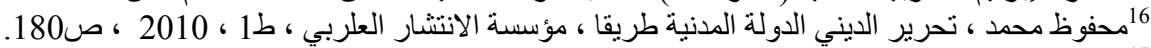

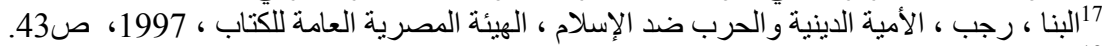

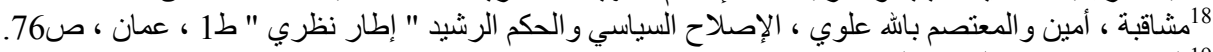

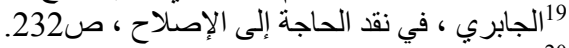

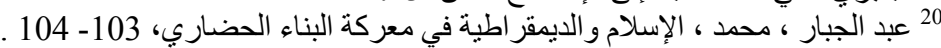

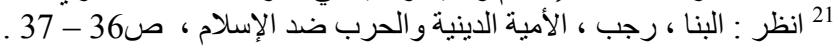

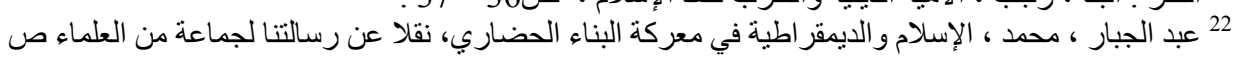




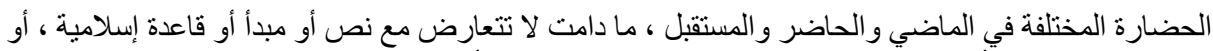

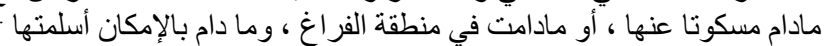

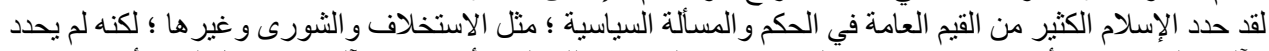

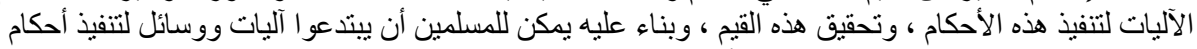

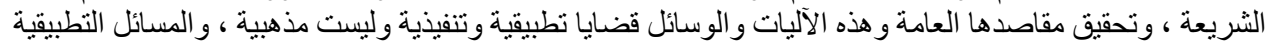

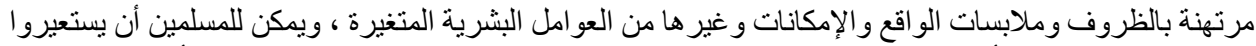

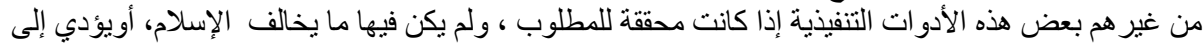

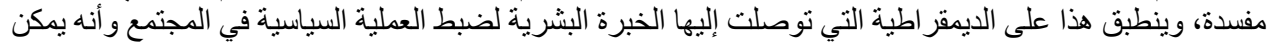

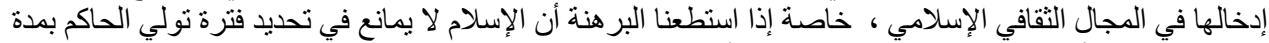

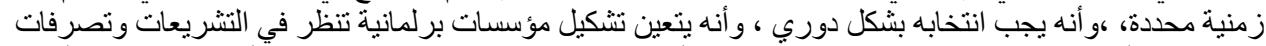

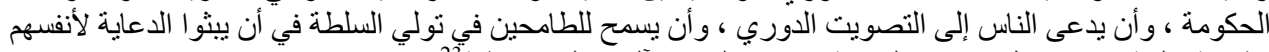

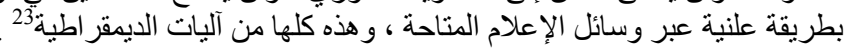

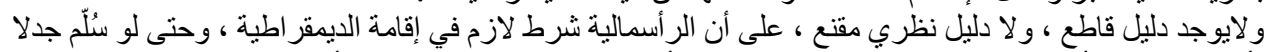

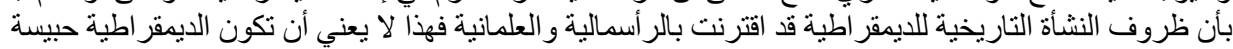

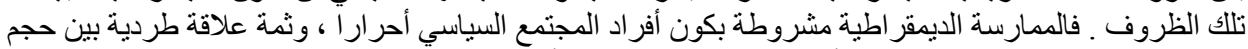

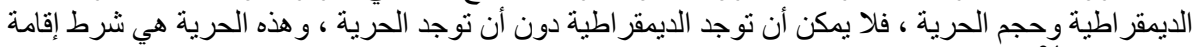
الديمقر اطية الدئن

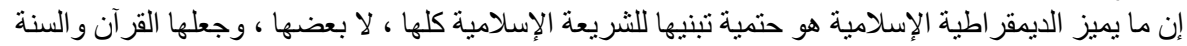

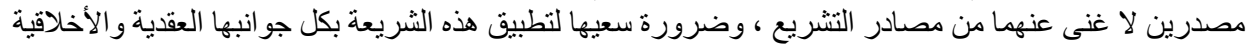

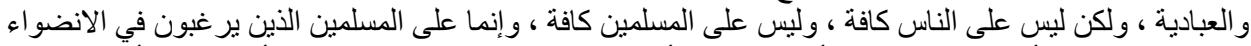

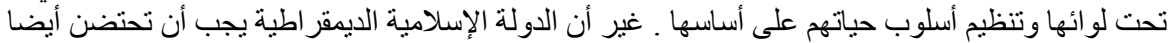

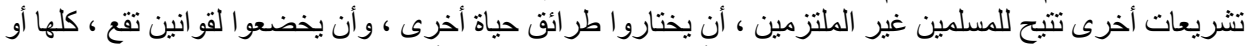

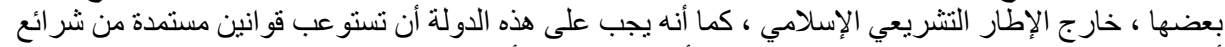

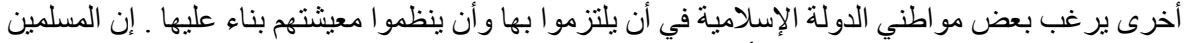

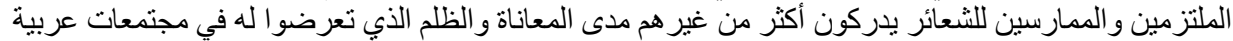

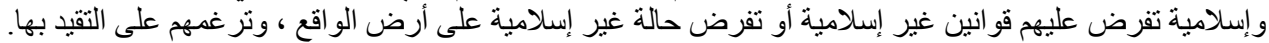

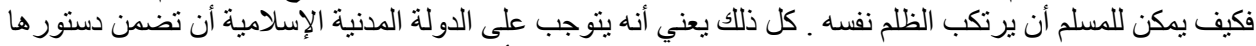

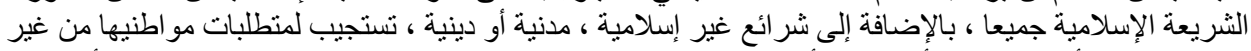

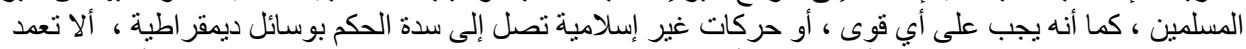

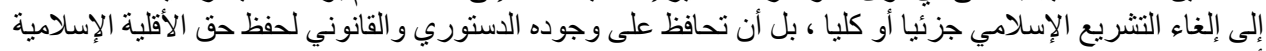

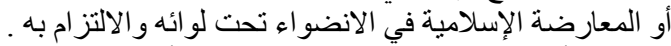

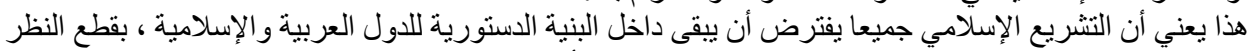

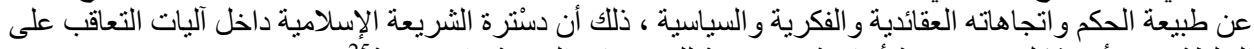

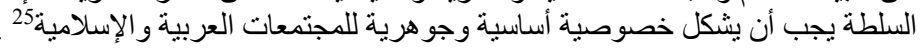
المطلب الثالث : الدولة المدنية و العلمانية.

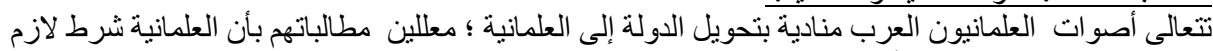

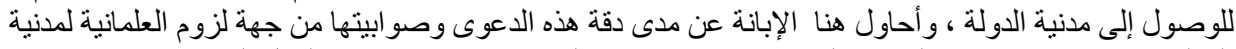

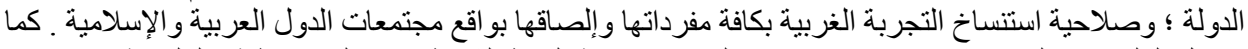

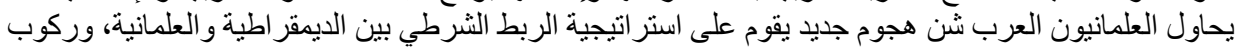

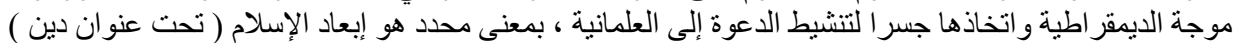

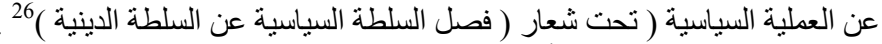

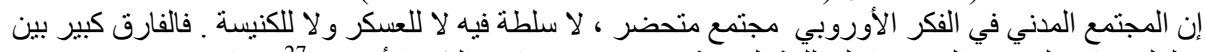

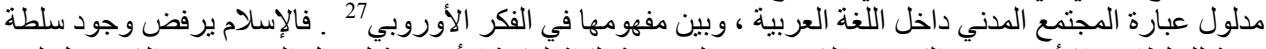

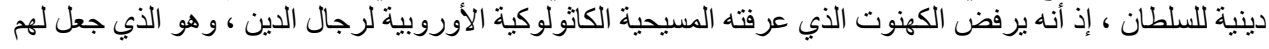

24 انظر : المرجع السابق ،ص : 24

24

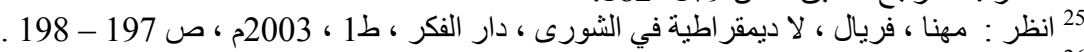

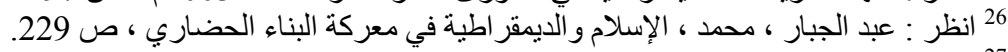




\section{Civil state and its impact on reform}

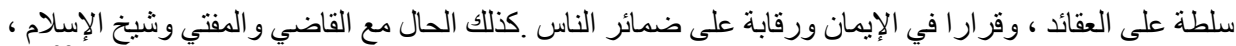

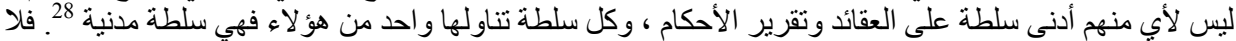

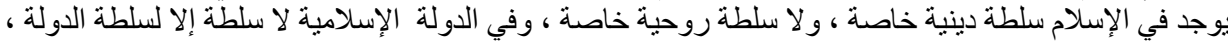

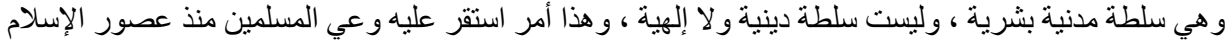

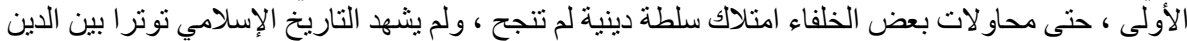

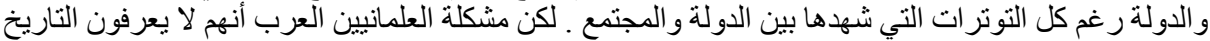

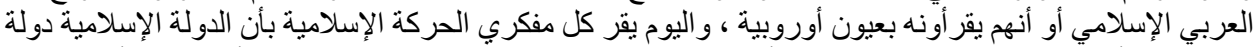

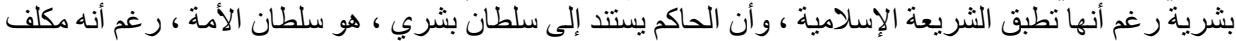
من بين جملة أمور أخرى ، بتطبيق أحكام الثريعة الإسلامية

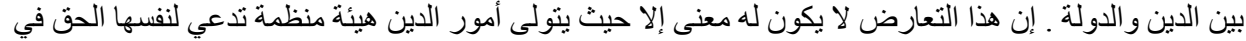

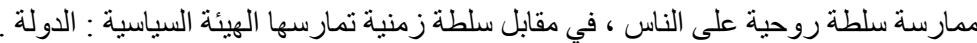

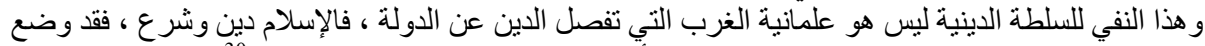

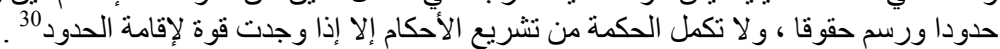

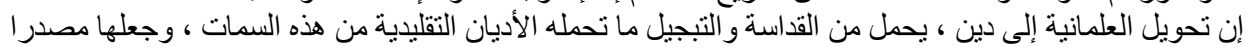

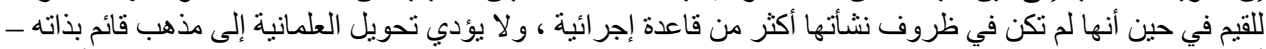

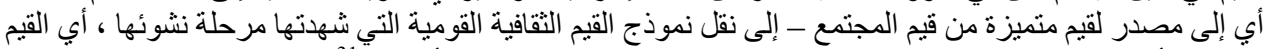

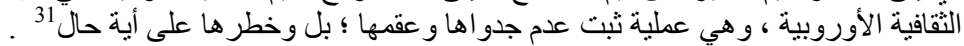

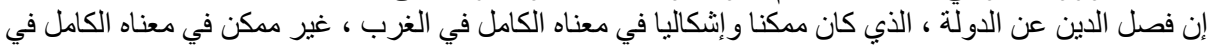

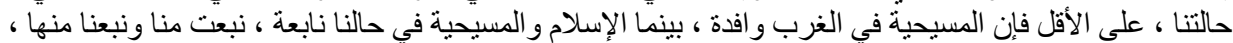

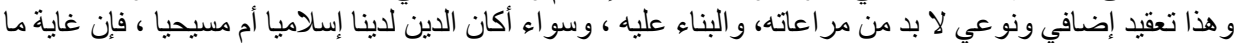

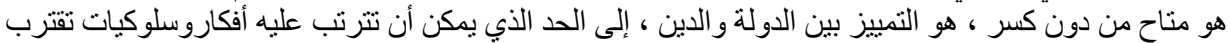

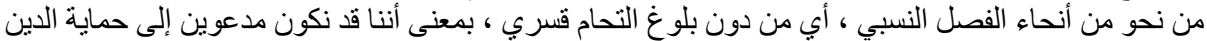

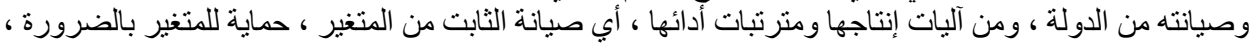

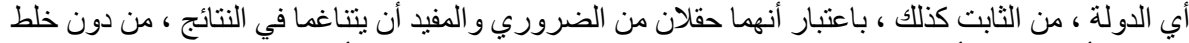

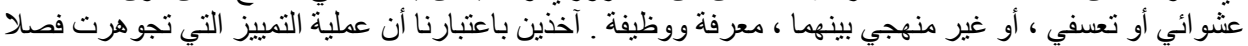

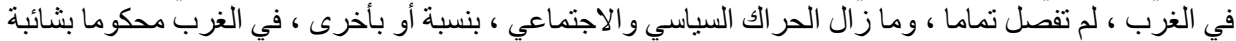

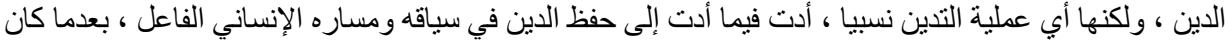

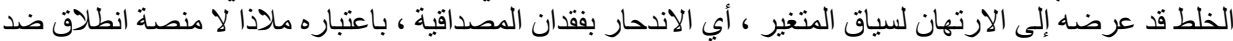

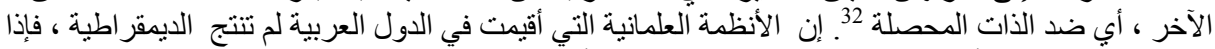

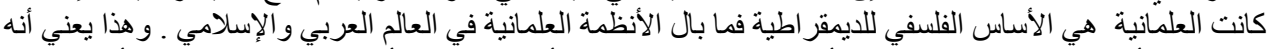

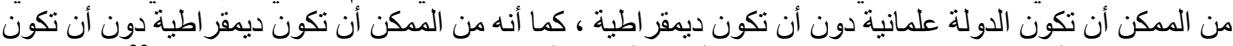

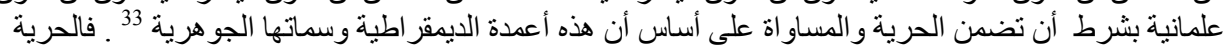

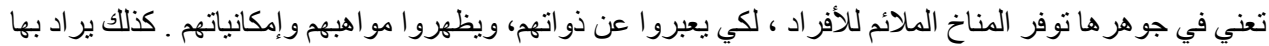

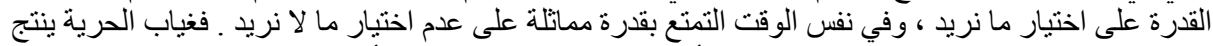

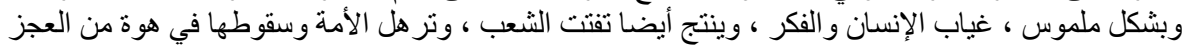

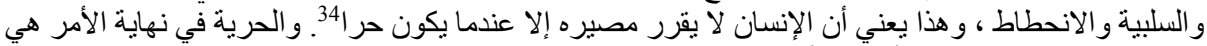

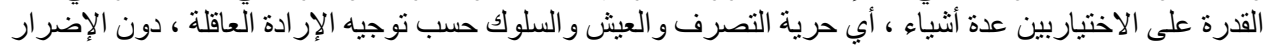

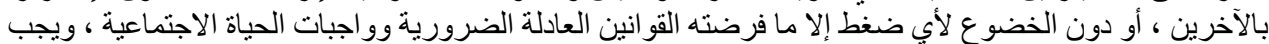

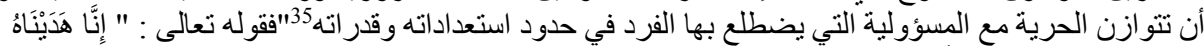

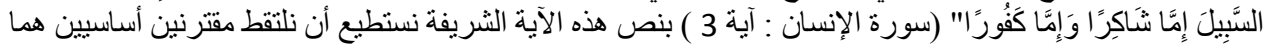

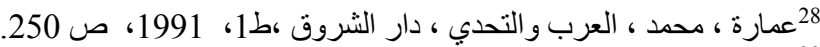

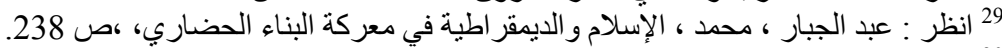

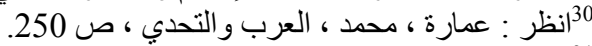

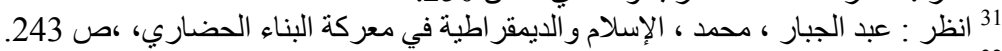

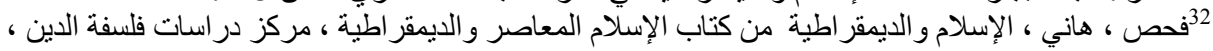

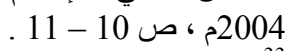

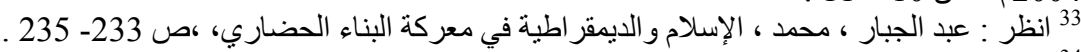

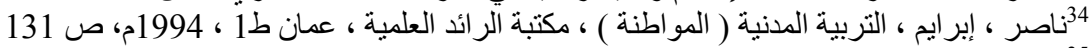

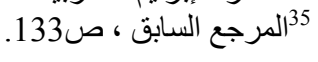


1ـ الحرية قيمة موضو عية ، يمكن أن تتحقق في العالم الخارجي ، ولو لم يكن ذلك ممكنا لم يُطلق الله تعالى

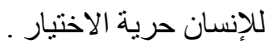

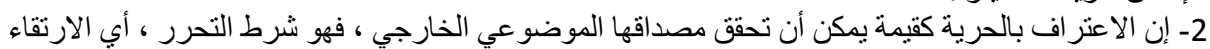

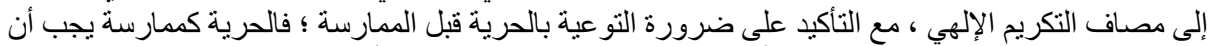

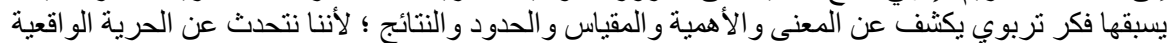

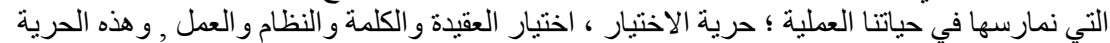

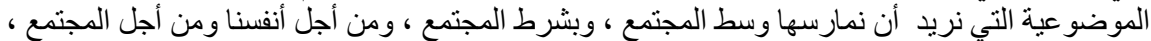

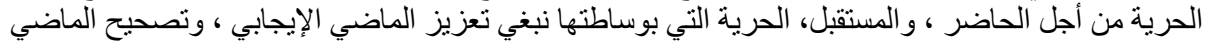

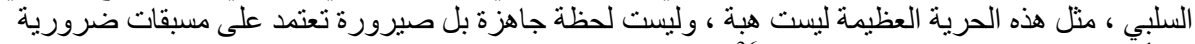

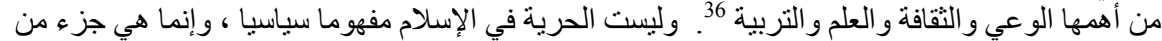

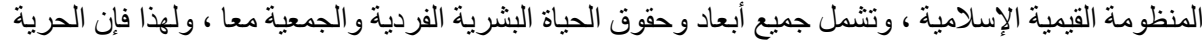

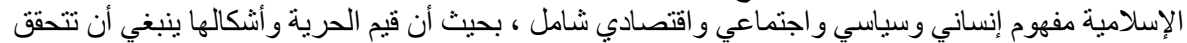

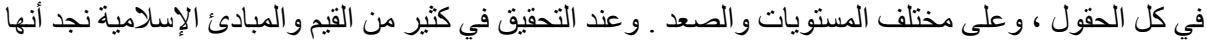

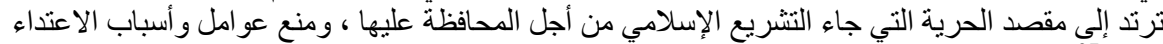

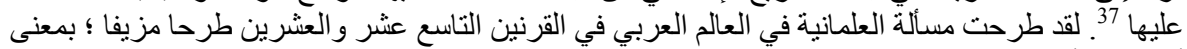

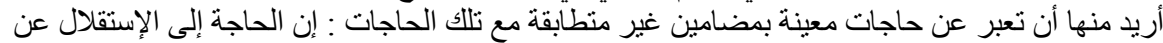

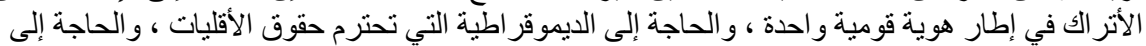

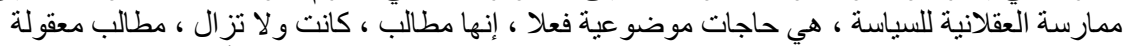

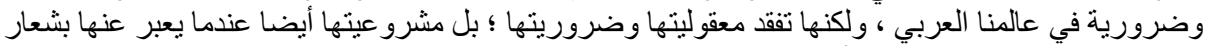

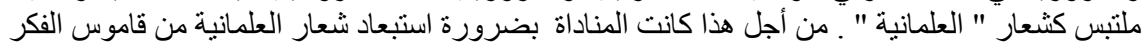

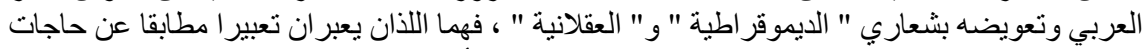

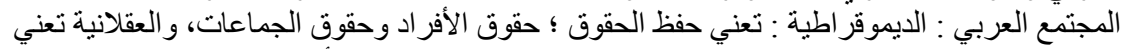

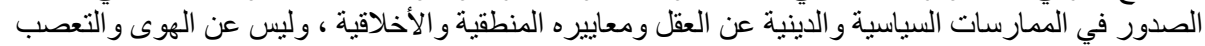

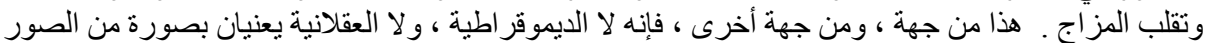

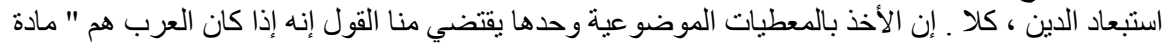

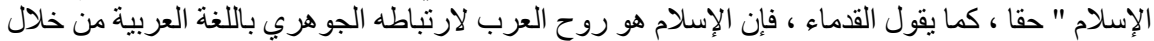

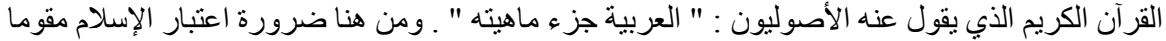

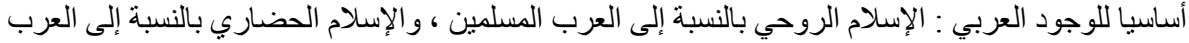

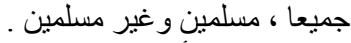

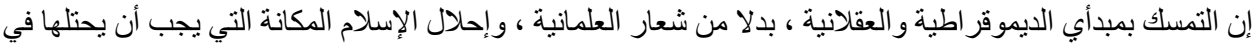

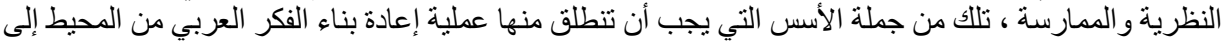

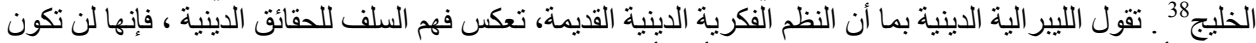

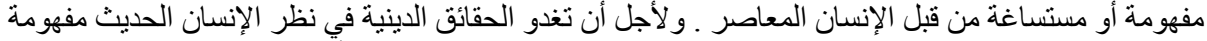

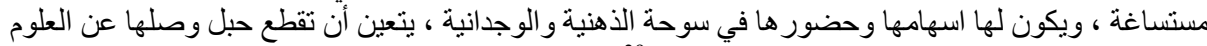

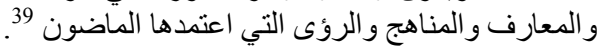

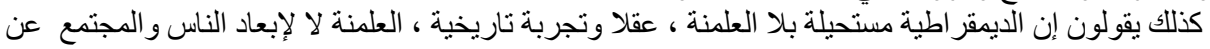

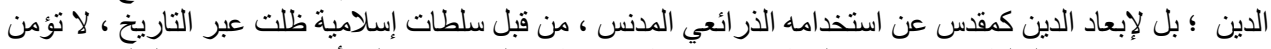

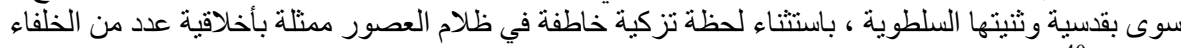
الر اشدين بقدئ.

185/نظر : الثابندر ، غالب ، أسس أولية لتعزيز الحرية الإسلامية ، من كتاب الإسلام المعاصر و الديمقر اطية ، ص $186-185$ 37 محفوظ محمد ، تحرير الديني الدولة الدننية طريقا ، مؤسسة الانتشار العلربي ، ط1 ، 85 ، 2010 ، ص 39 - 40 . 38

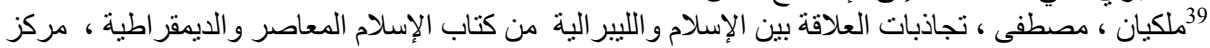

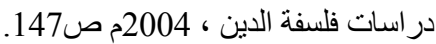
40 عيد ، عبد الرزاق ، الديمقراطية بين العلمانية والإسلام ، دار الفكر ، ط1 ، 1999م ، ص221. 


\section{Civil state and its impact on reform}

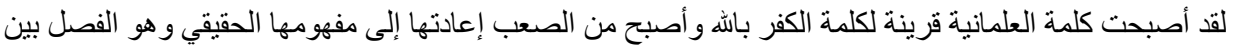

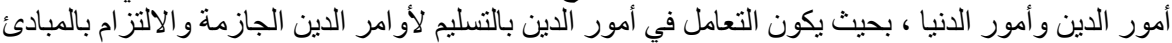

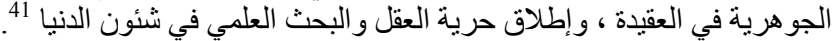
المطلب الرابع : الدولة الدئنة المافية في الو اقع الإسلامي.

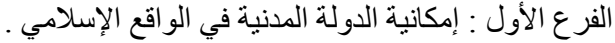

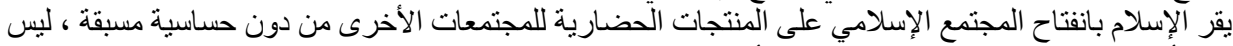

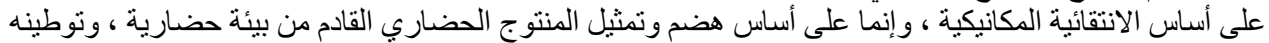

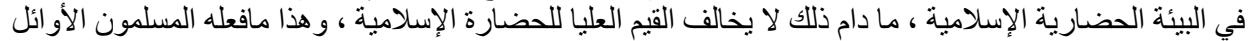

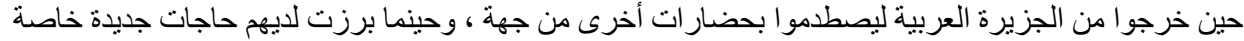

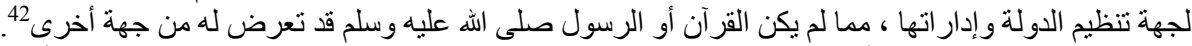

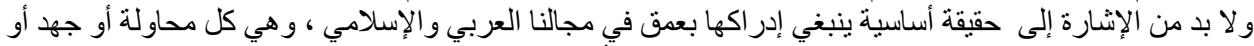

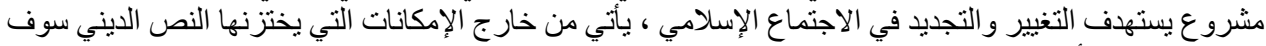

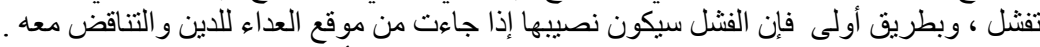

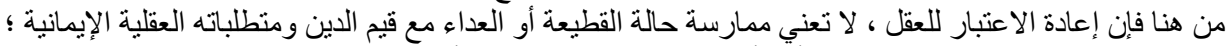

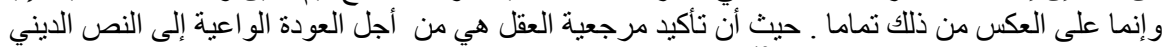

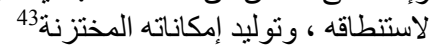

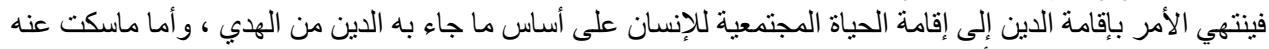

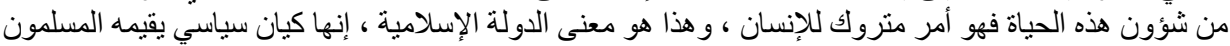

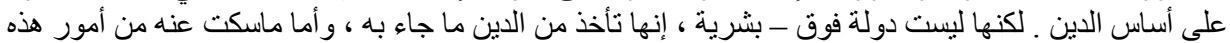

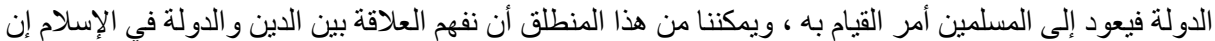

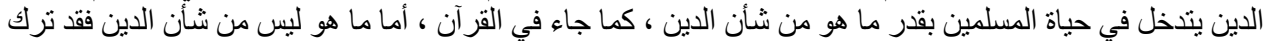

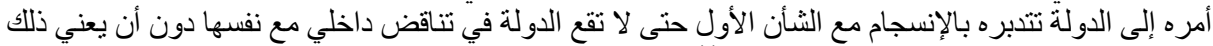

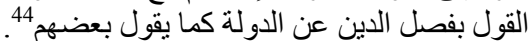

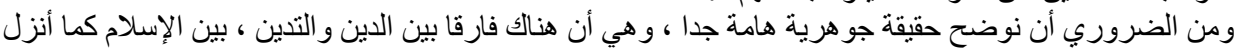

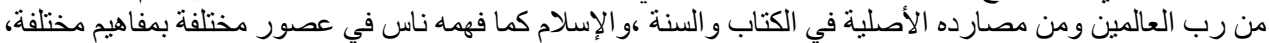

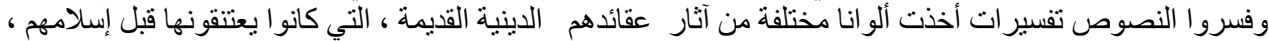

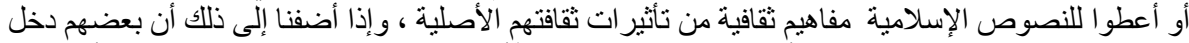

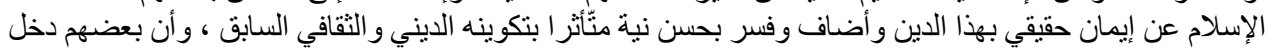

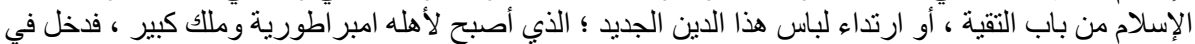

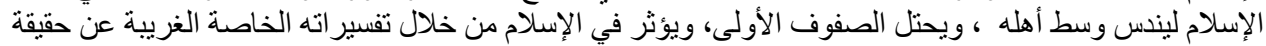

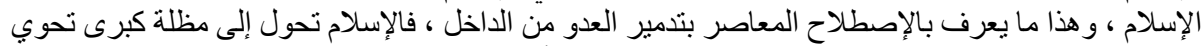

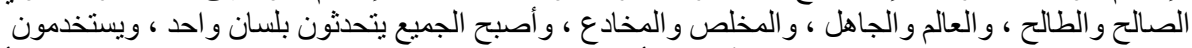

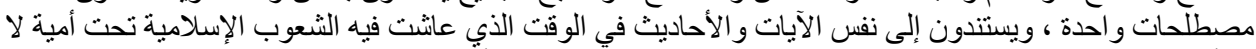

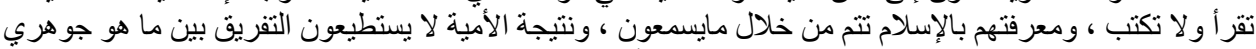

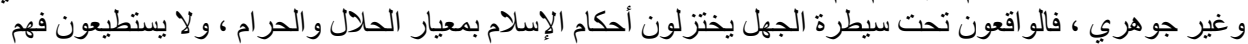

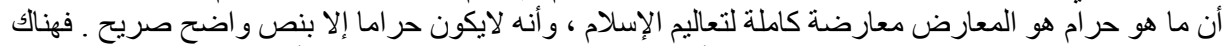

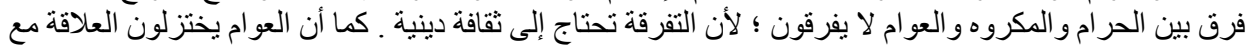

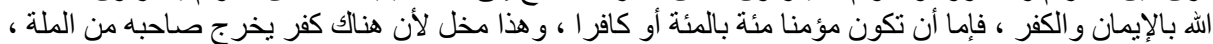

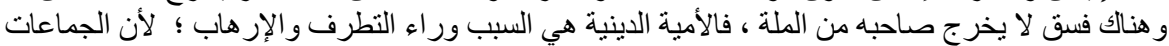

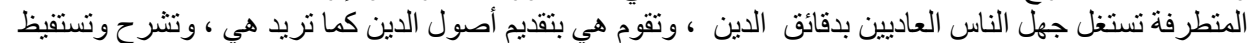

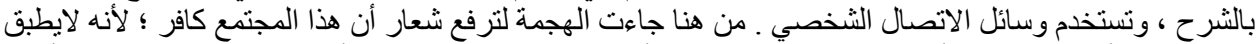

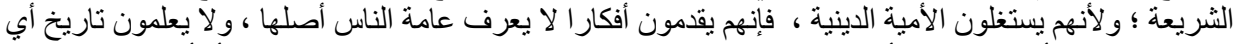

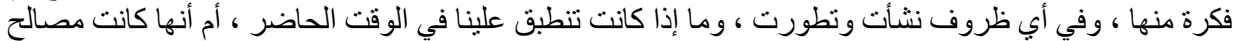

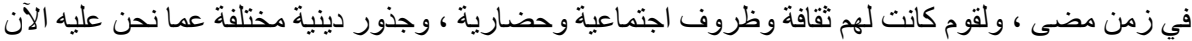
ونتيجة للأمية الدينية لا يعرف الأميون أن المسلم الحق يجب عليه ألا بسار عاع بالحكم على أحد بالكفر لأن الإيمان الان

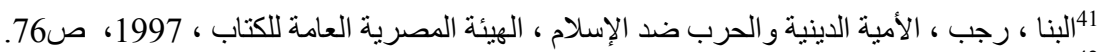

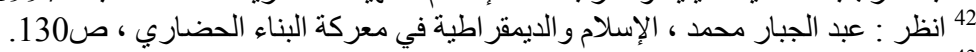

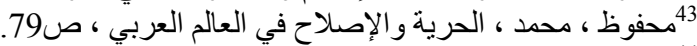

44 انظر : عبد الجبار ، محمد ، الإسلام و الديمقر اطية في معركة البناء الحضاري، ،ص 189- 191 


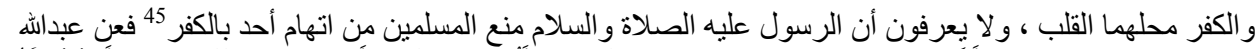

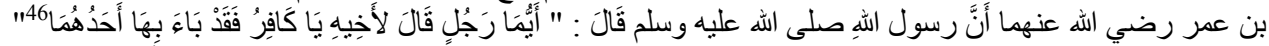

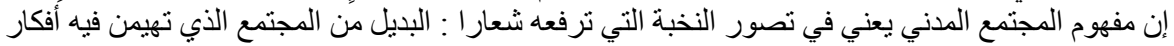

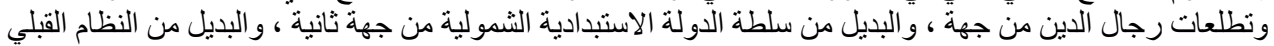

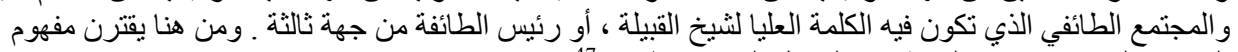

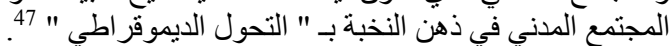

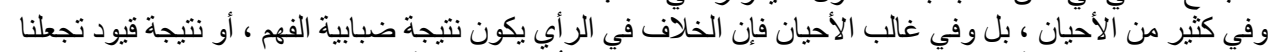

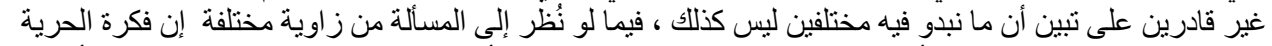

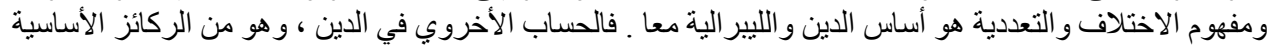

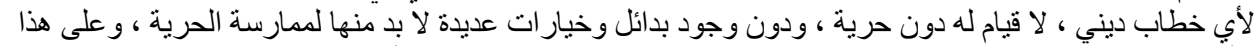

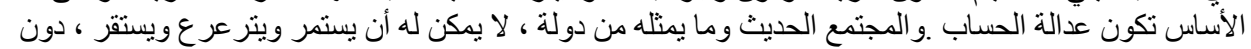

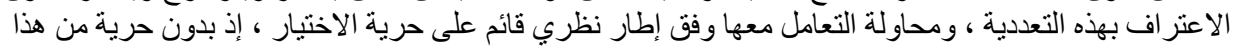

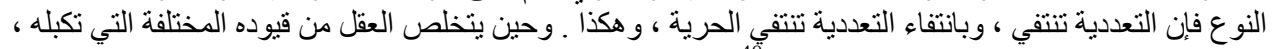

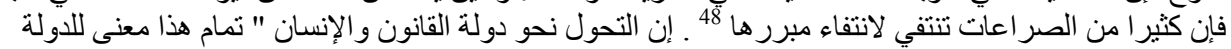

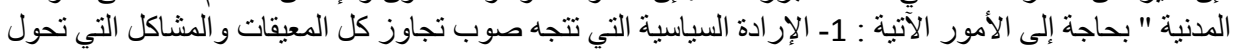

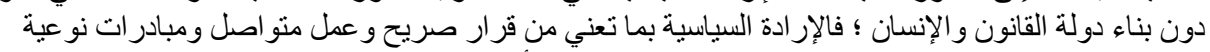

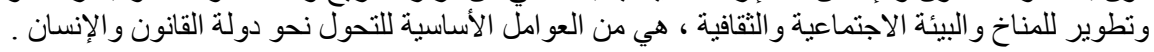

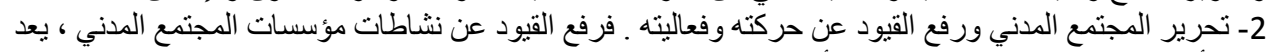

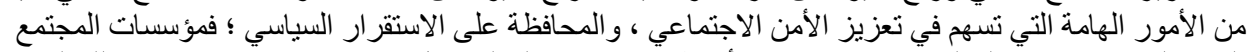

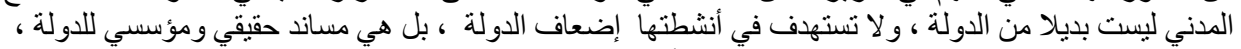

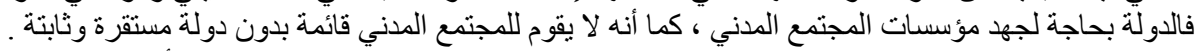

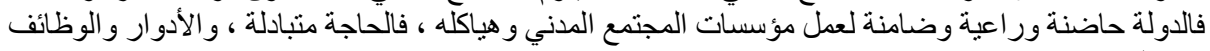
متكاملة.

فالدولة التي لا تحترم حقوق الإنسان ، وتتجاوز الدستور و القانون لأتفه الأسباب ، هي الدولة التي أخفقت في

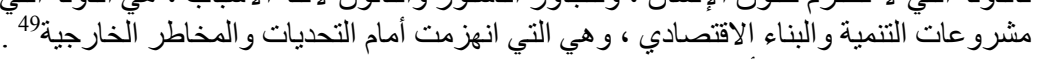
الفر ع الثاني : الإصلاح و أثنره في الدولة الأنة الدذنية.

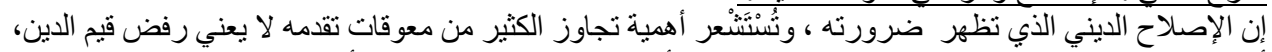

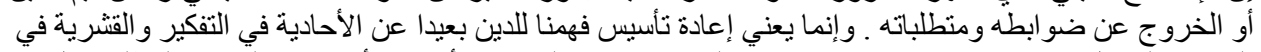

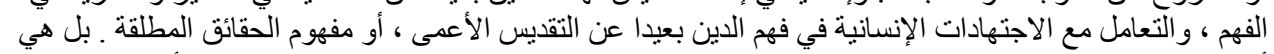

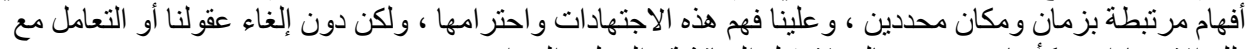

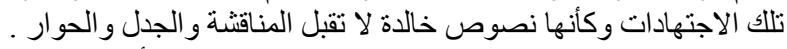

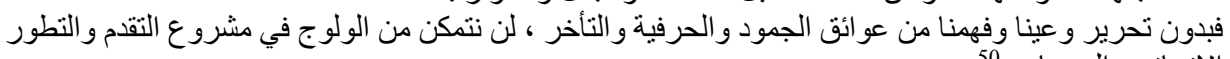

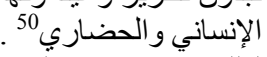

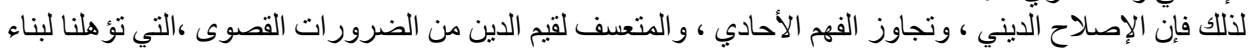

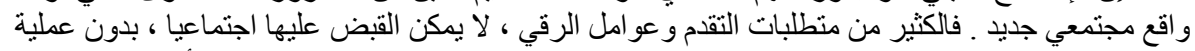

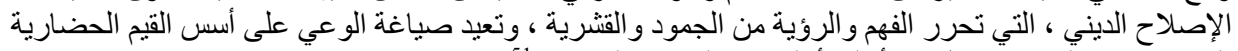

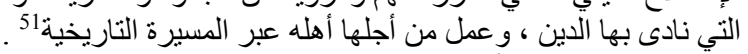

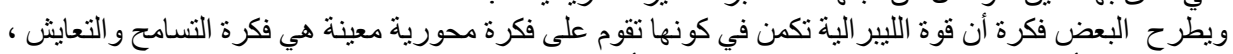

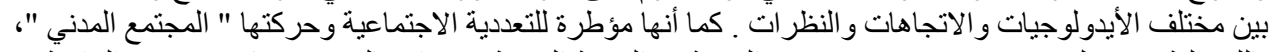

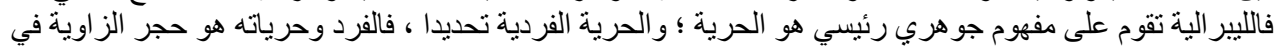

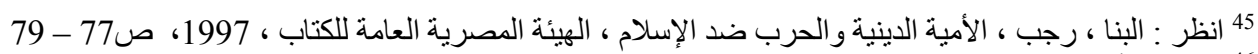

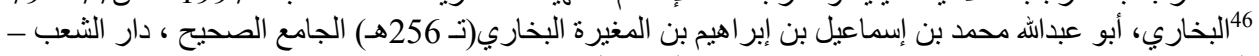

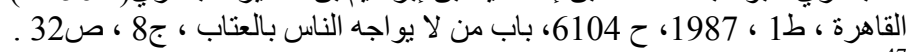

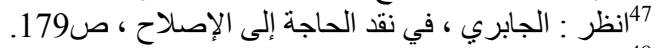

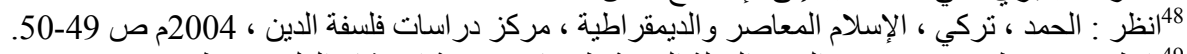

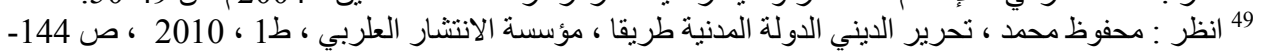

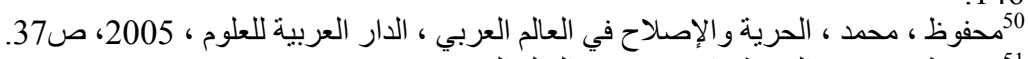

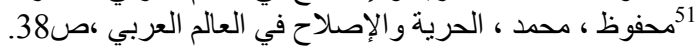




\section{Civil state and its impact on reform}

البناء الليير الي ـ و الحديث عن الفرد وحرياته وحقوقه يقود إلى الحديث عن مفهوم الدولة الحديثة في مقابل الدولة

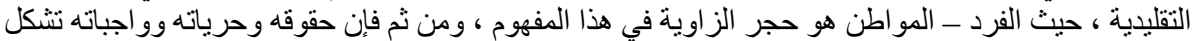

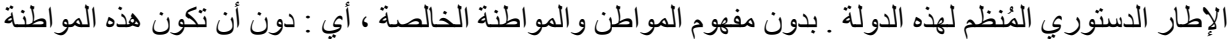

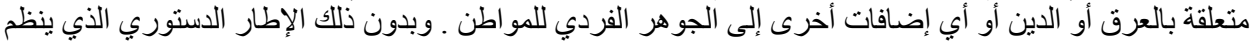

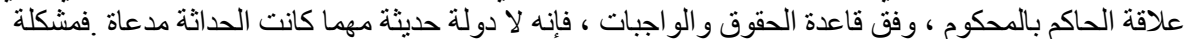

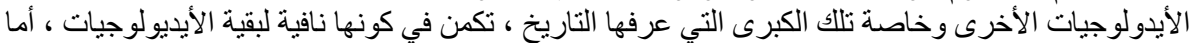

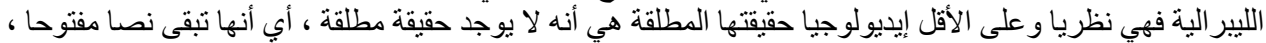

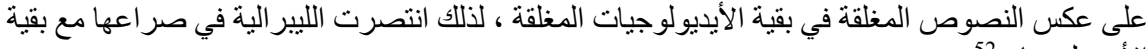

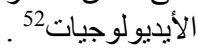

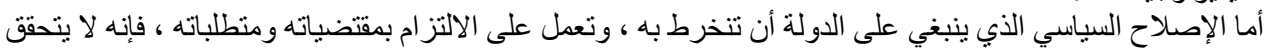

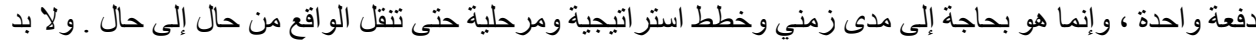

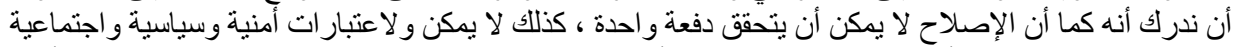

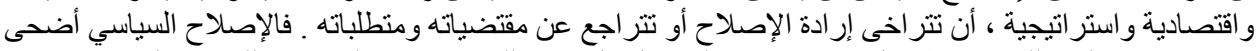

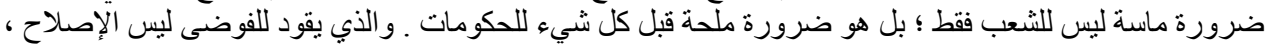

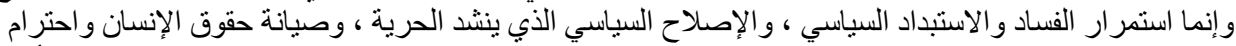

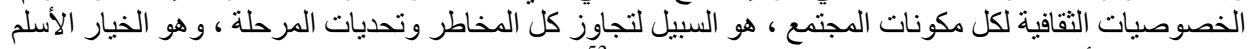

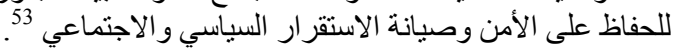

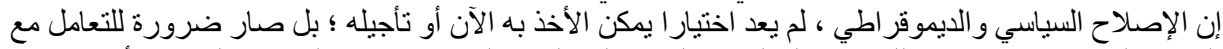

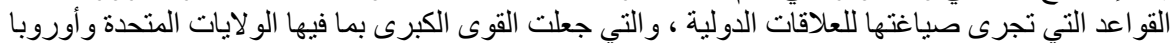

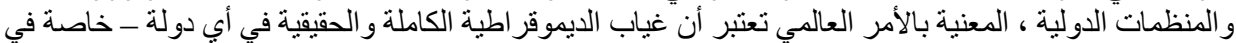

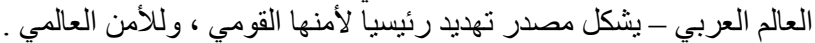

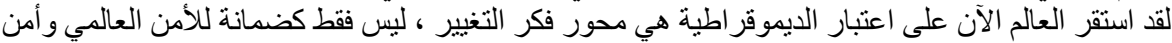

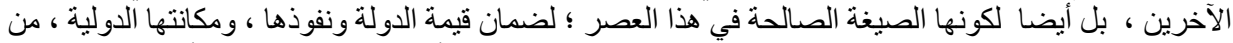

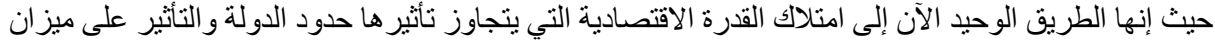

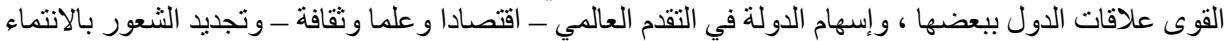

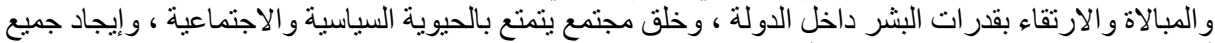

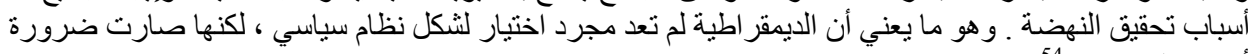

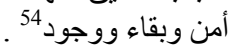

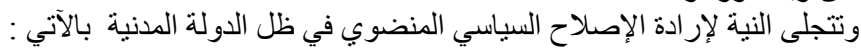

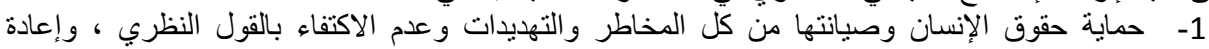

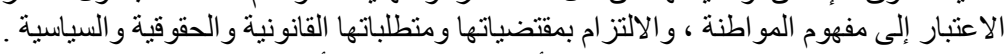

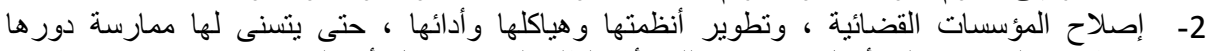

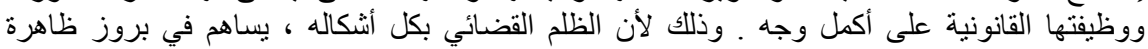

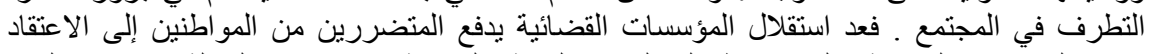

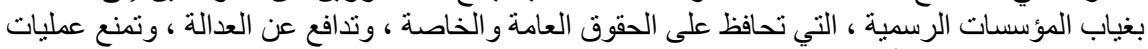

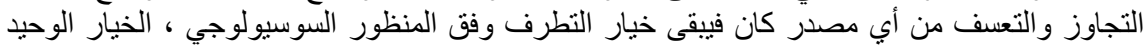

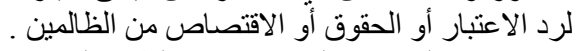

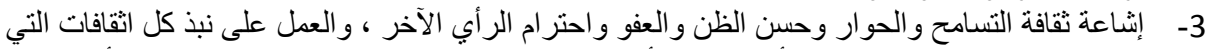

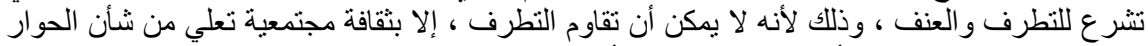

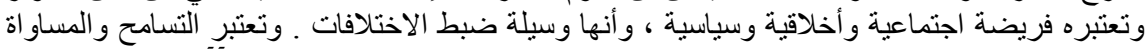

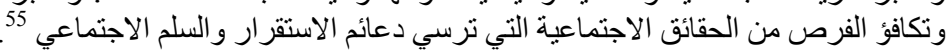

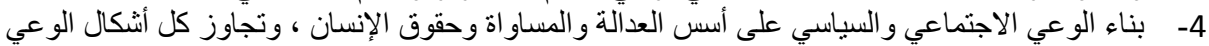

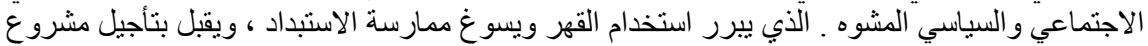

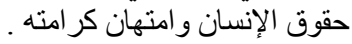

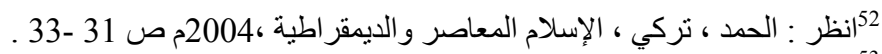

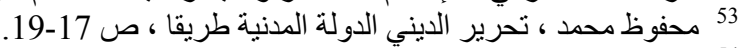

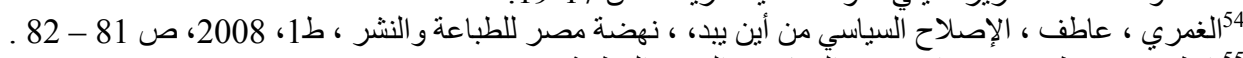

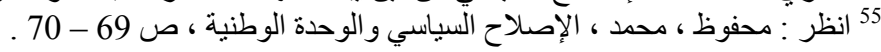




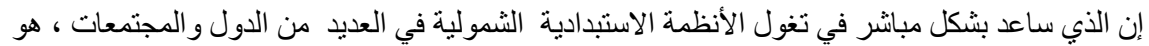

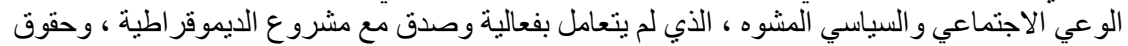

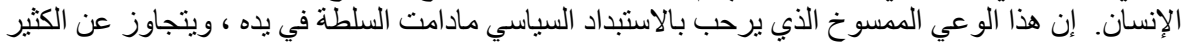

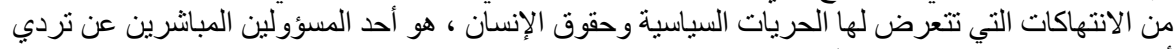

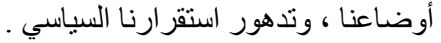

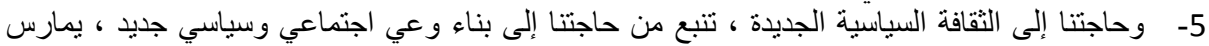

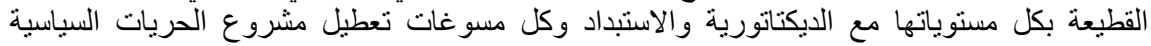

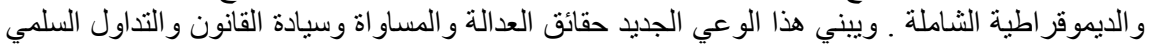
اللسلطة واحتر ام حق التعبير والاختلاف ، و وصياغة الو اقع كله وفق مقتضيات الديموقر اطية وحقوق الإنسان الخاتمة وفيها أهم

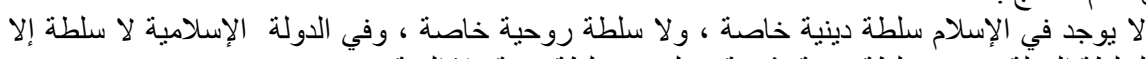

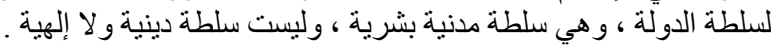

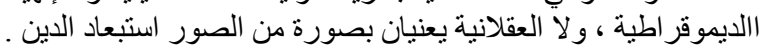

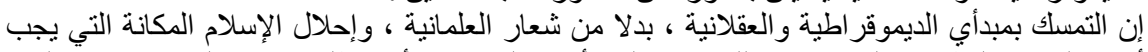

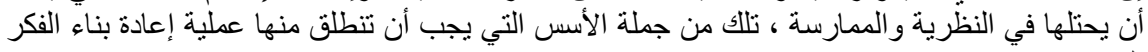

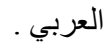
يقر الإسلام بانفتاح المجتمع الإسلامي على المنتجات الحضارية للمجتمعات الأخرى من دون حساسية

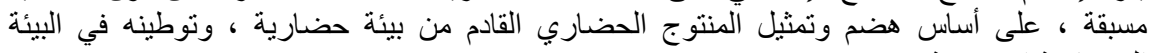

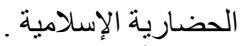

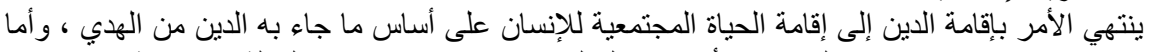

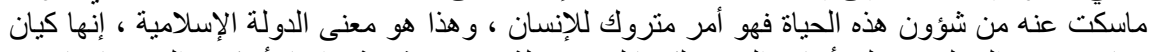

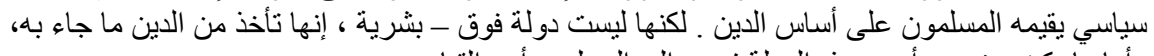

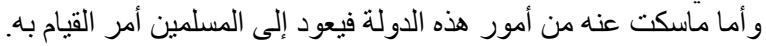

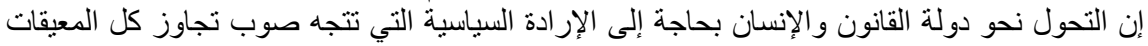

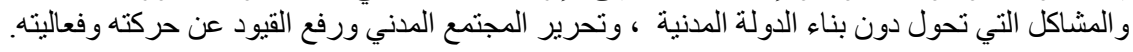

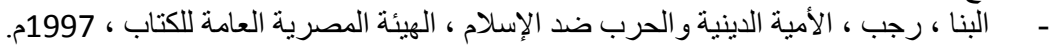

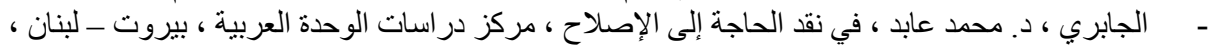
2005

حبيب ، كمال السعيد ، الأقليات و السياسة في الخبرة الإسلامية من بداية الدولة النبوية حتى نهاية الدولة

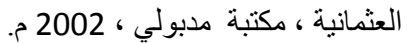

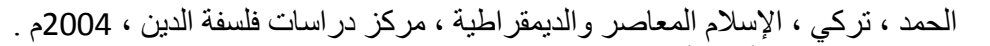

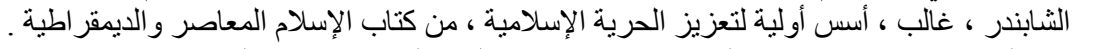

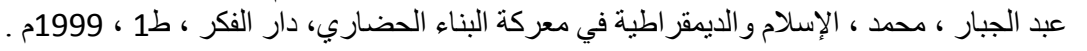

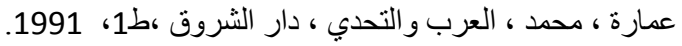

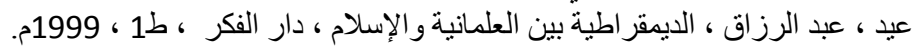

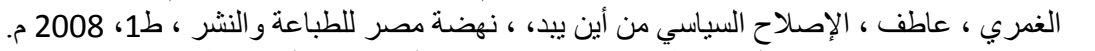

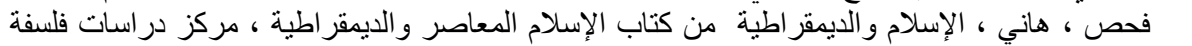

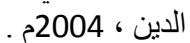

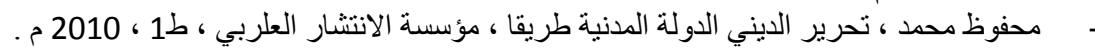

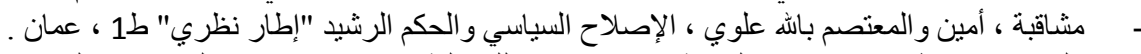

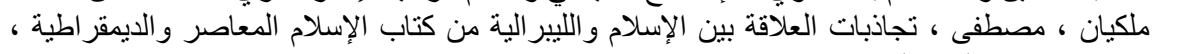

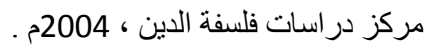

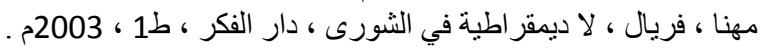
ناصر ، إبرايم ، التربية المدنية ( المواطنة ) ، دارية ، مكتبة الر ائد العلمية ، عمان ط1 ، 1994م. 


\section{References}

Abdel Jabbar, Mohammad, Islam and Democracy in the Battle of Civilizational Construction ar., Dar El-Fikr, 1st ed., 1999.

Al-Banna, Rajab, Religious Illiteracy and the War on Islam ar., General Egyptian Book Organization, 1997.

Al-Ghamri, Atef, Political Reform, Whence does it Start ar., NahdatMisr for Printing and Publishing, 1st ed., 2008.

Al-Hamad, Turki, Modern Islam and Democracy ar., Centre of the Philosophy of Religious Studies, 2004.

Al-Jabiri, Dr. Mohammad Abed, Critique of the Need for Reform ar., Centre for Arab Unity Studies, Beirut-Lebanon, 2005.

Al-Shabandar, Ghaleb, Basic Foundations for Promoting Islamic Freedom, from the book entitled "Contemporary Islamic and Democracy".

Amarah, Mohammad, the Arabs and the Challenge, Dar Al-Shurouq, 1st ed., 1991.

Eid, Abdel Razzaq, Democracy Between Secularism and Islam ar., Dar El-Fikr, 1st ed., 1999.

Fahas, Hani, Islam and Democracy, from the book entitled Modern Islam and Democracy, Centre of the Philosophy of Religious Studies, 2004.

Habib, Kamal Al-Said, Minorities and Politics in the Islamic Experience from the Establishment of the Prophetic State until the Demise of the Ottoman State ar., Madbouly Bookshop, 2002.

Mahfoudh Mohammad, Religious Liberation, State of Medina as a Path, Mu`assassat AlIntishar Al-Arabi, 1st ed., 2010.

Malkyan, Mustafa, The Push and Pull between Islam and Liberalism ar. from the book entitled Contemporary Islam and Democracy, Center of the Philosophy of Religious Studies, 2004.

Mashaqbeh, Amin and Al-Mu 'tassimBillah Alawi, Political Reform and Good Governance, a Theoretical Framework, 1st ed., Amman.

Mhanna, Firyal, No Democracy in Shura ar., Dar El-Fikr, 1st ed., 2003.

Nasser, Ibrahim, Civic Education ar. (Citizenship), Al-Raed Scientific Library, Amman 1st ed., 1994. 